\title{
A MASS SENSOR BASED ON PARAMETRIC RESONANCE
}

\author{
Wenhua Zhang and Kimberly L. Turner \\ Department of Mechanical and Environmental Engineering, University of California \\ Santa Barbara, CA 93106
}

\begin{abstract}
We present a parametric resonance-based mass sensor, comprised of a single-crystal silicon micro-oscillator with sensitivity at the pico-gram $\left(10^{-12} \mathrm{~g}\right)$ level when operating in air. This mass sensor detects mass change by measuring frequency shift at the boundary of the first order parametric resonance 'tongue'. High sensitivity is achievable due to the sharp jump in amplitude caused by parametric resonance at predictable drive frequencies. Pt deposition using focused ion beam and water vapor are used to change the mass of the testing oscillator. The results show that the sensitivity can be $>1$ order higher than the same oscillator working at simple harmonic resonance mode. The effect of noise on mass sensing ability in this nonlinear dynamic system is considered as well.
\end{abstract}

\section{INTRODUCTION}

The concept of tracking resonant frequency shifts of micro/nano-oscillators in simple harmonic resonance mode to measure mass change is a well-established technology in applications of chemical and biological sensing. In a microcantilever array, information on cantilever resonance frequency shifts can be used for recognition of a variety of chemical substances, such as water, primary alcohols, and alkanes [1]. A single cell with an estimated mass of about 0.7 pico-gram has been detected based on a micro-cantilever [2]. By creating even smaller oscillators, such as in nano scale, the ability of detecting femtograms or even attograms of mass change should be achievable [3].

One of the factors which limit the sensitivity of the simple harmonic resonance (SHR) based mass sensors is the quality factor $(Q)$. Micro/nano-oscillator with high $Q$ can detect small mass changes in the system because of the ability to resolve small frequency shift. Very low pressure and temperature is desired in many of these high-sensitive mass sensors, which makes the applications very limited. The sensitivity of mass detection can be dramatically lowered when operating in air or water, where $Q$ can be less than 10 .

In previous work, we have reported the concept of mass sensing using parametric resonance phenomenon $[\mathbf{4 , 5}$. This mass sensor detects mass change by measuring frequency shift of the stability boundary of the first order parametric resonance tongue $[4,5]$. This transition at the boundary is very sharp, thereby making small frequency changes easily detectable and the resolution high $[\mathbf{5}, \mathbf{6}]$. Since quality factor will not affect the sharpness of the transition, very small mass change can be detected in high pressure, even in air.

In this work, we present the first results on a mass sensor based on parametric resonance phenomenon. This preliminary device has sensitivity at the pico-gram $\left(10^{-12} \mathrm{~g}\right)$ level when

Acknowledgement: Travel support has been generously provided by the Transducers Research Foundation and by the DARPA MEMS and DARPA BioFlips programs. operating in air. This mass sensor is comprised of a single crystal silicon micro-oscillator (Fig.1), in which the backbone is supported by four folded beams to provide recovery force for the oscillation and driven by a set of non-interdigitated comb-fingers using fringing-field electrostatic force. Parametric resonance can be activated at certain frequencies because the non-interdigitated comb-fingers tune the stiffness of the oscillator [5]. To 'create' mass change to test the concept, Platinum is deposited on the backbone using Focused Ion Beam (FIB). A similar microoscillator is designed as a reference in the same package to improve the sensitivity of mass sensing through common-mode rejection.

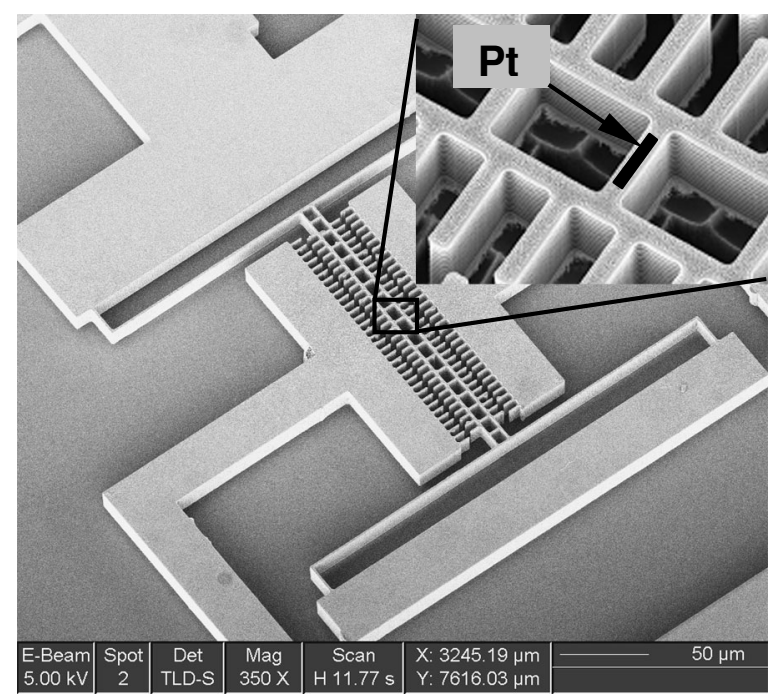

Figure 1. A SEM picture of the mass sensor. It has a backbone, 4 springs with folded beams to provide recovery force and one set of non-interdigitated comb-fingers to drive the oscillator. Pt deposition to change mass is schematically shown in this picture.

A noise analysis has been completed to determine the effect of noise on the device sensitivity. Because of the nonlinear dynamics nature of parametric resonance, the noise mechanism differs from normal simple harmonic resonance based mass sensors. Thermal noise, Brownian motion and actuation voltage fluctuation have been considered here. Both the experiment and analysis show that Brownian motion is the main noise source in mass sensing application of parametric resonance in air.

The ultimate sensitivity of the conceptual mass sensor is studied by testing water vapor content change in the environment. Less than $1 \mathrm{pg}$ of mass change in the oscillator has been detected in air. Significant improvements of this technology are achievable by reducing the size of the structure. This is discussed later in the paper.

\section{THEORY}

In previous work, we have discussed the parametric resonance dynamics of a similar oscillator in detail $[5,7]$. The 
micro-oscillator, shown in Fig.1, can be simplified as a massspring system with electrostatic force as the driving force. When electrical signal is applied on the non-interdigitated comb-fingers, the electrostatic force generated is dependent on the position of the oscillator. In the experiments presented here, we use a square rooted AC voltage signal $\left(V_{A}(1+\cos 2 \omega t)^{1 / 2}\right)$ to isolate the parametric resonance from direct harmonic response [6]. The movement of the device is governed by nonlinear Mathieu equation [5].

$\frac{d^{2} x}{d \tau^{2}}+\alpha \frac{d x}{d \tau}+(\beta+2 \delta \cos 2 \tau) x+\left(\delta_{3}+\delta_{3}^{\prime} \cos 2 \tau\right) x^{3}=0$

where $\quad \alpha=\frac{2 c}{m \omega} \quad \beta=\frac{4\left(k_{1}+r_{1} V_{A}^{2}\right)}{m \omega^{2}} \quad \delta=\frac{2 r_{1} V_{A}^{2}}{m \omega^{2}}$

$$
\delta_{3}=\frac{4 k_{3}+4 r_{3} V_{A}^{2}}{m \omega^{2}} \quad \delta_{3}^{\prime}=\frac{4 r_{3} V_{A}^{2}}{m \omega^{2}}
$$

$m, k_{l}$ and $k_{3}$ are the mass, linear and cubic mechanical stiffness of the oscillator, $c$ is the damping coefficient, $r_{l}$ and $r_{3}$ are linear and cubic "electrostatic stiffness" and $\tau=\omega t$ is a normalized time [5].

Figure 2 schematically shows the dynamics of this nonlinear Mathieu equation in $\beta$ - $\delta$ plane defined above [5]. According to dynamic characteristics, $\beta$ - $\delta$ plane can be divided into three areas. Area II inside the "tongue" is the resonance area of the first order parametric resonance with one non-trivial solution, while area I and III are non-resonance area with one trivial solution in area I and One trivial solution plus one non-trivial solution in area III. The characteristics of solutions in each area are schematically shown in Fig.2 as well, where " $S$ " means stable and "U" means unstable. Here we only consider stable solution, since unstable solution cannot be observed experimentally. The dynamics can be more easily understand in an experimental frequency response as shown in Fig.3.

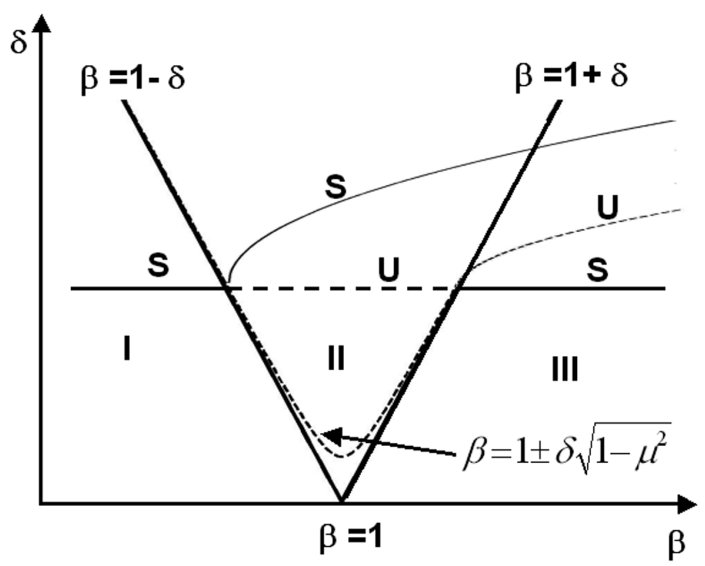

Figure 2. Dynamic characteristics of Nonlinear Mathieu Equation in the $\beta$ - $\delta$ plane. $\beta=1 \pm \delta$ are the transition curves, which divide $\beta$ - $\delta$ plane into Area I, II, III. Note the damping effects on transition curves and how the positions of the stable (dark trace) and unstable (broken trace) points vary as $\beta$ and $\delta$ are varied quasi-statically.

Figure 3 shows the typical frequency response of parametric resonance inside resonance area (area II) and outside of the resonance area (area I and III). As the driving frequency goes up, the amplitude of the oscillator increases from the left boundary of area II and keeps increasing past the boundary of area III. The frequency where the drop happens depends on initial condition of the frequency applied. When sweeping down the driving frequency from area III, movement of the oscillator jumps to a large value from zero at the right boundary of area II and reduces in amplitude thereafter until it reaches zero at the left boundary of area II. The frequency at the right boundary of area II, where the "jump" happens, is given by $f=\frac{1}{2 \pi} \sqrt{4 k+2 r V_{A}^{2} / m}$, where $k, m, V_{A}$ and $r$ are stiffness, mass, driving voltage amplitude applied and the coefficient of electrostatic force respectively. A small mass change $(\Delta m)$ in the oscillator causes this "jump" frequency to shift $(\Delta f)$. Therefore, this mass change can be detected by measuring the frequency shift. Figure 4 shows the mechanism of parametric resonance based mass sensor, where the mass change is given by $|\Delta m|-2 m|\Delta f| / f_{0}$.

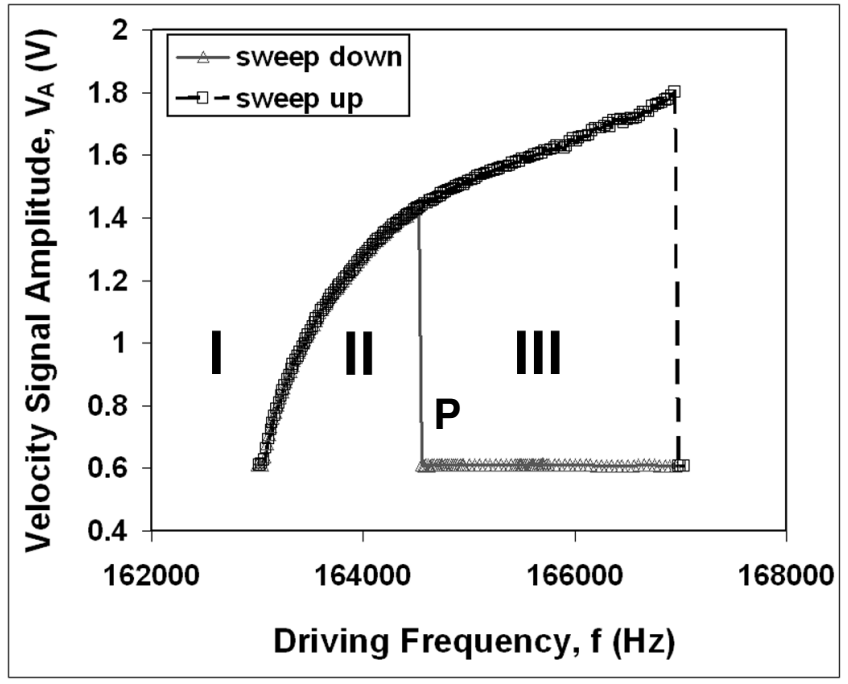

Figure 3. A frequency response curves of the first order parametric resonance inside and outside of stability region. Area II is inside of "tongue", as shown in Fig.2, while I and III are outside. When sweep frequency down, resonance only happens in area II and there is completely no movement in area I and III. A jump happens at the right boundary of area II.

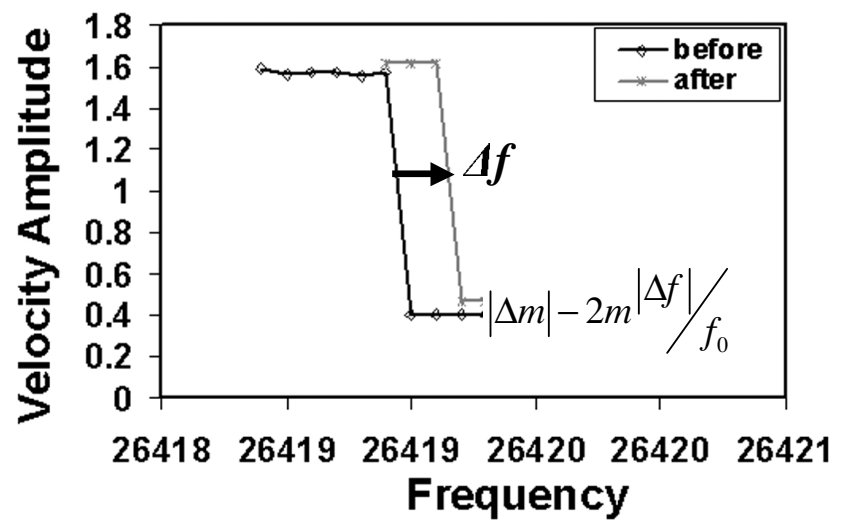

Figure 4. Schematic of the mechanism of parametric resonancebased mass sensor. By measuring the frequency shift at right side of area II (Fig.3), mass change in the oscillator can be found.

The sensitivity of parametric resonance based mass sensor depends on the smallest frequency shift we can measure. Since the "jump" is a characteristic of nonlinear dynamics, the detectable frequency shift can be very small. In our previous work, a $0.001 \mathrm{~Hz}$ 
frequency shift has been observed [6]. Damping affects the resonance boundary of the tongue, as shown in Fig.2. At high damping, for example in air, actuation of parametric resonance requires more energy than at low pressure. However, the characteristics of dynamics will not change. Therefore, damping has little effect on the sensitivity of parametric resonance based mass sensors.

\section{EXPERIMENT}

\section{DEVICE FABRICATION}

The micro-oscillators are fabricated from SOI wafer with highly doped device layer. After patterning, a deep silicon etch process, $\mathrm{BOSCH}$, is performed to form the micro structure, following by removal of photo resist left and wet chemical release of the buried silicon oxide layer to form the suspended MEMS structure. The thickness of the device is about $18 \mu \mathrm{m}$, which is defined by the thickness of the top device layer. The fabricated oscillator is a single crystal silicon device and electrically conductive.

Eight devices with different dimensions and configurations have been made. Two of them are tested here, named as device\#3 and device \#4. The two devices have the same dimensions, except that the springs of device\#3 are of $1.5 \mu \mathrm{m}$ wide and that of device \#4 are of $2.0 \mu \mathrm{m}$ wide. The backbone mass of the two devices is about $30 \mathrm{ng}$ and the natural frequencies are about $49 \mathrm{kHz}$ and 83 $\mathrm{kHz}$.

\section{MASS SENSING WITH Pt DEPOSITION}

As s proof of concept, we change the mass of the oscillator by attaching a set volume of Platinum $(\mathrm{Pt})$ on device\#3 using a FIB (Focused Ion Beam), as shown in Fig.1. Three such mass changes, about 1, 4 and $0.4 \mu \mathrm{m}^{3} \mathrm{Pt}$ deposition (corresponding to about 20, 80 and $8 \mathrm{pg}$ ), have been made. We record the frequency change at the right boundary of the first order parametric resonance area as discussed earlier before and after the deposition in air using laser vibrometry [8]. Meanwhile, device\#4 works as a reference sensor and the frequency information is also recorded as a reference to environmental fluctuation, such as humidity and temperature.

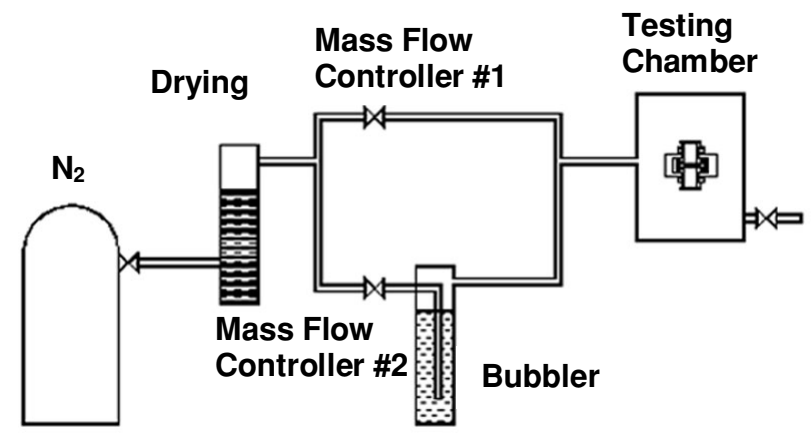

Figure 5. Gas handling setup

\section{MASS SENSING WITH WATER VAPOR}

To perform a more sensitive test, the mass sensor was also tested using adsorption of water vapor. A gas handling setup is built to control the water vapor content in testing chamber, as shown in Fig.5. A dry nitrogen gas flows through mass flow controller \#1 directly to the testing chamber and another dry $\mathrm{N}_{2}$ through mass flow controller \#2 with water vapor from the water bubbler. By setting the flow rates of the two mass flow controllers, relative water content can be adjusted. The frequency information of device \#4 at the right boundary of the first order parametric resonance area is recorded, as water content in the testing chamber is adjusted. Because of the native silicon oxide on the surface of the oscillator [9], water molecules are absorbed on the surface and the mass of the oscillator is changed. By measuring this frequency shift, mass change can be determined.

\section{RESULTS AND DISCUSSION}

The results of frequency shift in Pt deposition test are shown in Fig.6. After each mass change, the "jump" frequency is recorded many times in a few days. The results are rectified according to frequency change in reference oscillator. As shown in Fig.6, the average frequency shift is about 36, 151 and $17 \mathrm{~Hz}$, corresponding to 1,4 and $0.4 \mu \mathrm{m} \mathrm{Pt}$ deposition. Good correlation is shown in these tests between the volume of Pt and "jump" frequency shift, as we discussed earlier, mass change is linearly dependent on frequency shift, $|\Delta m|-2 m|\Delta f| / f_{0}$, when mass change in the oscillator is small. The minimum mass change here is about $8 \mathrm{pg}$.

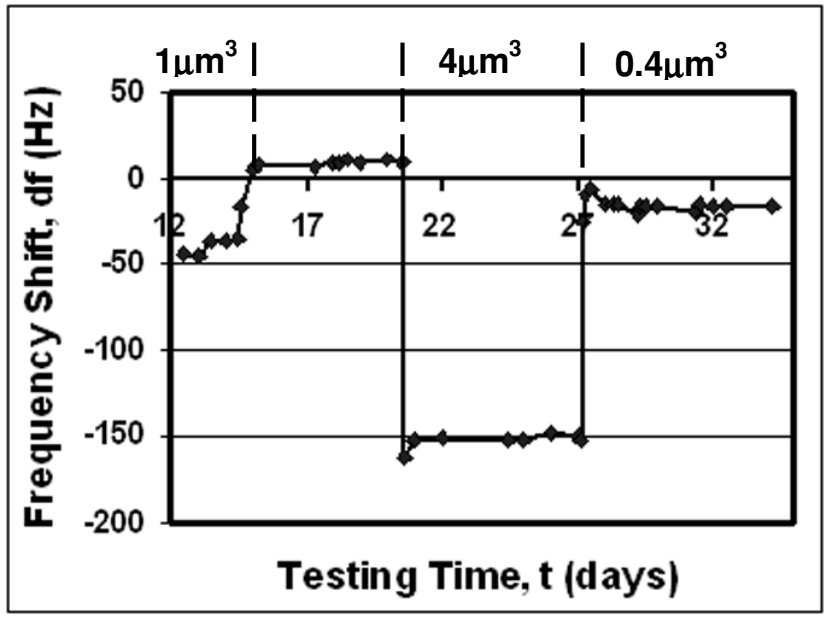

Figure 6. Frequency shift in Pt deposition tests. Three mass changes have been made, 1,4 and $0.4 \mu^{3}$ corresponding to about 20,80 and $8 \mathrm{pg}$ respectively.

As with most micro-sensors [10-12], noise is an important issue in mass sensing using parametric resonance technology. The ability to detect ultra-fine frequency shift because of the nature of parametric resonance is compromised by noise problems in the oscillator. As we mentioned earlier, a $0.001 \mathrm{~Hz}$ or even smaller frequency shift has been observed [6]. However, in our current experiment, the frequency fluctuation is much larger than this value and the standard deviation of this frequency fluctuation we measured in these two oscillators is about $0.8 \mathrm{~Hz}$ at room temperature. To analyze noise problems in mass sensing, we consider thermal noise, Brownian motion and Driving voltage fluctuation. Because of the complexity of parametric resonance, numerical simulation method is used to calculate the effects of these noise sources. Brownian motion of oscillators is attribute to be the main noise source in parametric resonance mass sensing. In room temperature, the standard deviation of Brownian motion is estimated about $0.5 \AA$, which brings about $0.7 \mathrm{~Hz}$ of frequency fluctuation. The result agrees well with measured result.

Certainly, there are other issues, which can be considered noise effects in mass sensing, such as frequency drift caused by humidity fluctuation and temperature fluctuation in the 
environment. We notice that noise caused by this frequency drift can be larger than that caused by Brownian motion even with reference sensor when the test lasts days. These issues are currently being addressed.

To find the ultimate sensitivity of this mass sensor based on parametric resonance, we use the micro-oscillator to detect water content change in the testing chamber. By adjusting the water content, we observe the frequency shift simultaneously. Figure 7 shows the results of frequency shift of Device\#4 as the relative water content is changed in air. When water content is switched between $5 \%$ and $10 \%$ as shown in Fig. 7 from A-B, the rate of frequency shift changes. As water vapor is turn on and off from B$\mathrm{C}$, the results shows good consistency between frequency shift and water vapor content. The smallest controllable frequency shift as shown in Fig. 7 is about $2 \mathrm{~Hz}$, which is equivalent to mass change of $0.7 \mathrm{pg}$.

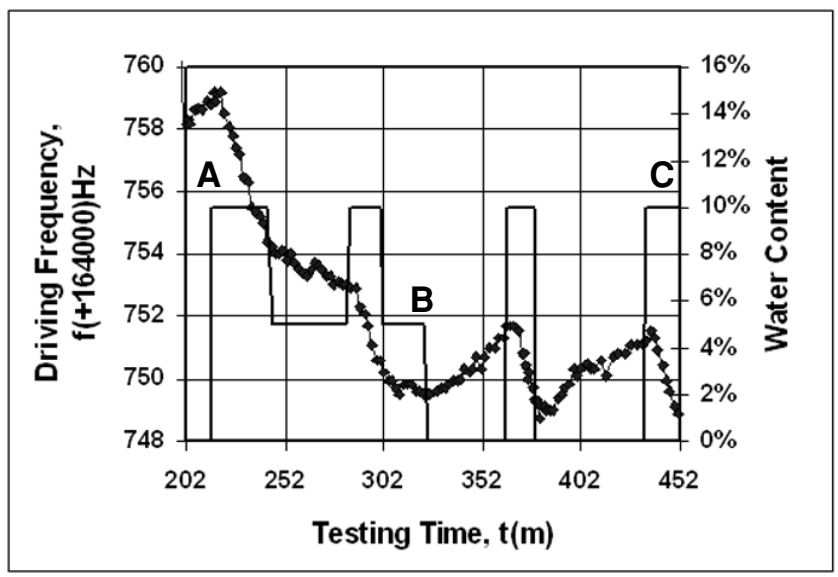

Figure 7. Frequency shifts at the right side of the first parametric resonance area as adjusting water content.

Because of damping effect, the sensitivity of simple harmonic resonance (SHR) based mass sensor decrease dramatically in air. It is difficult for the devices we tested here to resolve $100 \mathrm{~Hz}$ shift in SHR mode with Q about 50. Using parametric resonance, the sensitivity can improve more than 1 order of magnitude. Damping has little effect on the sensing ability when operated in a parametric resonance mode.

The devices are just prototype mass sensors to test the concept of parametric resonance mass sensing. By tailoring the oscillator dimensions (for example, the cantilever geometry), increasing resonance frequency, or designing oscillator arrays, the sensitivity can be further improved, thus extending their application to DNA, virus and other analytes with mass in the order of attograms. We calculated the noise level of device\#3 with dimensions reduced by a factor of 5 . The equivalent mass resolution will be at femto-gram level in air pressure. Now, many smaller micro/nano oscillators have been made with higher frequency, such as micro-cantilever and nanowire [13, 14], and parametric resonance has been realized in these devices. Thus, the possibility of significant improvements in cantilever based sensors is achievable, and currently underway.

\section{CONCLUSIONS}

Parametric resonance based mass sensor has been developed in this work. The measurement, taken in air, demonstrates that parametric resonance-based mass sensors can improve sensitivity 1-2 orders compared to the same sensor working in harmonic mode. The prototype mass sensors can detect mass changes at pico gram level when operating in parametric resonance mode. By decreasing the mass of oscillator and increasing resonance frequency, the sensitivity can be further improved.

\section{REFERENCES}

1. F.M. Battiston, J.P. Ramseyer, H.P. Lang, M.K. Baller, C. Gerber, J.K. Gimzewski, E. Meyer, and H.J. Guntherodt, "A chemical sensor based on a microfabricated cantilever array with simultaneous resonance-frequency and bending readout", Sensors \& Actuators B-Chemical, B77, 122 (2001).

2. B. Ilic, D. Czaplewski, M. Zalalutdinov, H.G. Craighead, P. Neuzil, C. Campagnolo, and C. Batt, "Single cell detection with micromechanical oscillators", Journal of Vacuum Science \& Technology B, 19, 2825 (2001).

3. Z.J. Davis, G. Abadal, O. Kuhn, O. Hansen, F. Grey, and A. Boisen, "Fabrication and characterization of nanoresonating devices for mass detection", J. Vac. Sci. Technol. B, Microelectron. Nanometer Struct., 18, 612 (2000).

4. K.L. Turner and W. Zhang, "Design and analysis of a dynamic MEM chemical sensor", Proceedings of the 2001 American Control Conference, Arlington, VA, USA, 25-27 June 2001, pp. 1214-1218.

5. W. Zhang, R. Baskaran, and K.L. Turner, "Effect of cubic nonlinearity on auto-parametrically amplified resonant MEMS mass sensor", Sens. Actuators A, Phys., 102/1-2, (2002).

6. K.L. Turner, P.G. Hartwell, F.M. Bertsch, and N.C. MacDonald, "Parametric resonance in a microelectromechanical torsional oscillator", ASME International Mechanical Engineering Congress and Exposition Proceedings of Microelectromechanical Systems (MEMS), Anaheim, CA, USA, 15-20 Nov. 1998, ASME, New York, NY, USA.(1998), pp. 335-340.

7. W. Zhang, R. Baskaran, and K.L. Turner, "Tuning the dynamic behavior of parametric resonance in a micromechanical oscillator", Appl. Phys. Lett., 82, 130 (2003).

8. K.L. Turner, "Multi-dimensional MEMS motion characterization using laser vibrometry", Transducers'99 The 10th International conference on solid-state Sensors and Actuators, Digest of Technical Papers, Sendai, Japan, 7-10 June 1999, pp. 1144-1147.

9. H.P. Lang, M.K. Baller, F.M. Battiston, J. Fritz, R. Berger, J.P. Ramseyer, P. Fornaro, E. Meyer, H.J. Guntherodt, J. Brugger, U. Drechsler, H. Rothuizen, M. Despont, P. Vettiger, C. Gerber, and J.K. Gimzewski, "The nanomechanical NOSE", Proceedings of 12th International Workshop on Micro Electro Mechanical Systems - MEMS, Orlando, FL, USA, 17-21 Jan. 1999, pp. 9-13.

10. A.N. Cleland and M.L. Roukes, "Fabrication of high frequency nanometer scale mechanical resonators from bulk Si crystals", Appl. Phys. Lett., 69, 2653 (1996).

11. J.R. Vig and K. Yoonkee, "Noise in microelectromechanical system resonators", IEEE Transactions on Ultrasonics Ferroelectrics \& Frequency Control, 46, 1558 (1999).

12. T.B. Gabrielson, "Mechanical-thermal noise in micromachined acoustic and vibration sensors", IEEE Trans. Electron Devices, 40, 903 (1993).

13. Y. Min-Feng, G.J. Wagner, R.S. Ruoff, and M.J. Dyer, "Realization of parametric resonances in a nanowire mechanical system with nanomanipulation inside a scanning electron microscope", Physical Review B-Condensed Matter, 66, 073406/1 (2002).

14. M. Napoli, R. Baskaran, K. Turner, and B. Bamieh, "Understanding mechanical domain parametric resonance in microcantilevers", Proceedings IEEE Sixteenth Annual International Conference on Micro Electro Mechanical Systems, Kyoto, Japan, 19-23 Jan. 2003, pp. 169-172. 